\title{
Lo mejor del Congreso 2018 de la Sociedad Europea de Cardiología
}

\author{
Dres. Yamel Ache, Agustina Bonino, Carlos Guamán, \\ Sebastián Lorenzo, Sofía Noria
}

\begin{abstract}
Resumen para la comunidad. vos para la mayoría de los pacientes.

Realizaremos un breve resumen de algunos de los principales trabajos científicos presentados:

- Coronary CT Angiography and 5-Year Risk of Myocardial Infarction: The SCOT-HEART Investigators.

- Partial Oral Treatment of Endocarditis: POET. GLOBAL LEADERS.

- One-Year Outcomes after PCI Strategies in Cardiogenic Shock: CULPRIT-SHOCK.

Palabras clave: $\quad$ PREVENCIÓN PRIMARIA

ANGIOGRAFÍA CORONARIA

ANGIOGRAFÍA POR TOMOGRAFÍA COMPUTARIZADA

INFARTO

ENDOCARDITIS

STENTS LIBERADORES DE FÁRMACOS

SHOCK CARDIOGÉNICO
\end{abstract}

El Congreso Europeo de Cardiología se llevó a cabo en la ciudad de Múnich del 25 al 29 de agosto. Sin duda, uno de los eventos más importantes e innovadores de la comunidad cardiológica reunió a más de 32.000 profesionales, con destacados invitados internacionales y múltiples actividades científicas. Además de las tradicionales presentaciones de estudios, se inauguró un área de Salud Digital: Digital Health Area donde se llevaron a cabo sesiones continuas sobre E-Cardiology, con múltiples presentaciones de apps móviles, telemedicina y tecnología digital tanto para los profesionales como

Se presentaron las nuevas guías europeas sobre Hipertensión Arterial, tan esperadas luego del debate planteado en relación con las guías americanas en 2017. Las pautas europeas se mantendrán dentro del umbral de diagnóstico de 140/90 mmHg y se centrarán en mejorar las tasas de control a través de combinaciones iniciales de dos fármacos antihipertensi-

- Use of aspirin to reduce risk of initial vascular events in patients at moderate risk of cardiovascular disease: ARRIVE.

- Ticagrelor plus aspirin for 1 month, followed by ticagrelor monotherapy for 23 months vs aspirin plus clopidogrel or ticagrelor for 12 months, followed by aspirin monotherapy for 12 months after implantation of a drug-eluting stent:

\section{The best of the 2018 European Society of Cardiology Congress}

\section{Summary}

The European Congress of Cardiology was held at Munich from August $25^{\text {th }}$ to $29^{\text {th }}$. Undoubtedly one of the most important and innovative events of the cardiology community, it brought together more than 32.000 professionals, with prominent international guests and multiple scientific activities. In addition to the traditional presentations of studies, ESC 2018 inaugurated a: Digital Health Area, where continuous sessions on E-Cardiology were held with multiple presentations of mobile apps, telemedicine and digital technology for both, the professionals and the community.

The new european guidelines on Arterial Hypertension, awaited after the discussion related with the American guidelines in 2017, were presented. European blood pressure guidelines will stick with the $140 / 90 \mathrm{mmHg}$ diagnostic threshold and will focus on improving control rates through initial two-drug antihypertensive combinations for most patients. We will make a brief summary of some of the main scientific trials presented:

- Use of aspirin to reduce risk of initial vascular events in patients at moderate risk of cardiovascular disease: ARRIVE. - Coronary CT Angiography and 5-Year Risk of Myocardial Infarction: The SCOT-HEART Investigators.

Consejo Editorial de la Revista Uruguaya de Cardiología

Correspondencia: Dra. Yamel Ache. Correo electrónico: yamelache@gmail.com

Los autores declaran no tener conflictos de intereses.

Recibido 2 Oct, 2018; aceptado 17 Oct, 2018. 
- Partial Oral Treatment of Endocarditis: POET.

- Ticagrelor plus aspirin for 1 month, followed by ticagrelor monotherapy for 23 months vs aspirin plus clopidogrel or ticagrelor for 12 months, followed by aspirin monotherapy for 12 months after implantation of a drug-eluting stent: GLOBAL LEADERS.

- One-Year Outcomes after PCI Strategies in Cardiogenic Shock: CULPRIT-SHOCK

Key words: $\quad$ PRIMARY PREVENTION

CORONARY ANGIOGRAPHY

COMPUTED TOMOGRAPHY ANGIOGRAPHY

INFARCTION

ENDOCARDITIS

DRUG- ELUTING STENTS

CARDIOGENIC, SHOCK

\section{Use of aspirin to reduce risk of initial vascular events in patients at moderate risk of cardiovascular disease: ARRIVE}

El uso de aspirina en la prevención primaria de eventos cardiovasculares (CV) continúa siendo controvertido. Con el objetivo de valorar su eficacia y seguridad en pacientes con riesgo CV moderado, se realizó el estudio ARRIVE(1).

Es un estudio aleatorizado, doble ciego, placebo-control, multicéntrico, en el que participaron 501 centros de siete países. Se incluyeron hombres mayores de 55 años y mujeres mayores de 60 años, con riesgo CV moderado, definido como el riesgo de enfermedad a 10 años del $10 \%$ a $20 \%$, los cuales fueron asignados a recibir diariamente $100 \mathrm{mg}$ de aspirina con cubierta entérica o placebo. Se excluyeron los pacientes que presentaban un riesgo de sangrado alto o diabetes. El objetivo primario de eficacia fue valorar el tiempo hasta la primera aparición del compuesto muerte CV, infarto agudo de miocardio (IAM), accidente cerebrovascular (ACV), angina inestable (AI) y accidente isquémico transitorio (AIT). El objetivo en cuanto a seguridad fue evaluar eventos hemorrágicos $\mathrm{u}$ otros efectos adversos. El diseño del estudio fue por intención de tratar. Participaron 12.546 pacientes: 6.270 recibieron aspirina y 6.276 placebo, con una media de seguimiento de 60 meses $^{(1)}$.

Los resultados fueron presentados por el profesor Michael Gaziano, autor principal del estudio y publicados simultáneamente en la revista The Lancet. En el análisis por intención de tratar, no hubo diferencias significativas en el objetivo primario entre ambos grupos: 269 pacientes $(4,29 \%)$ en el grupo que recibió aspirina versus 281 pacientes $(4,48 \%)$ en el grupo placebo (HR0,96; IC95\%: 0,81-1,13; p=0,6038)(1). En el análisis por protocolo tampoco se observaron diferencias significativas en el objetivo primario, sin embargo cuando se consideran los componentes individuales como el riesgo de IAM total y no mortal se redujo con la aspirina (HR0,53; IC95\%: 0,36-0,79; $\mathrm{p}=0,0014$ y HR0,55; IC95\%: 0,36-0,84; $\mathrm{p}=0,0056$, respectivamente) $)^{(1,2)}$.
Con respecto al objetivo secundario, las hemorragias gastrointestinales (la mayoría leves) fueron dos veces más frecuentes en el grupo de aspirina que en el grupo placebo $(0,97 \%$ frente a $0,46 \%$, HR2,11; IC95\%: 1,36-3,28; $\mathrm{p}=0,0007)$, pero no hubo diferencias en los eventos hemorrágicos fatales entre los grupos ${ }^{(1,2)}$. La incidencia general de eventos adversos relacionados con el tratamiento fue menor en los que recibían placebo $(13,54 \%$ vs aspirina $16,75 \%, \mathrm{p}<0,0001)^{(1)}$. Se documentaron 321 muertes durante el seguimiento, con una diferencia no significativa entre los grupos $(n=160$ [2,55\%] en el grupo de aspirina y $\mathrm{n}=161[2,57 \%]$ en el grupo placebo; $p=0,94)$. De esas muertes, 108 se debieron a IAM, ACV u otra causa vascular fatal $^{(1)}$

La tasa de eventos fue mucho menor de lo esperado, lo que hace que el estudio sea más representativo de una población de bajo riesgo. Esto puede deberse a que los pacientes recibían medicación para controlar los factores de riesgo ${ }^{(1,2)}$. El papel de la aspirina en prevención primaria de pacientes con riesgo moderado continúa sin poder definirse. Los hallazgos con respecto a los efectos de la aspirina son más consistentes con los observados previamente en los estudios de prevención primaria de bajo riesgo ${ }^{(3)}$. Como dato de interés adicional, no se observaron efectos significativos a corto plazo con respecto a prevención del cáncer. A propósito de este aspecto, Gaziano consideró que "el tiempo de seguimiento fue insuficiente para evaluar resultados a largo plazo"(2).

Al finalizar la presentación el profesor Gaziano concluyó: "El uso de aspirina sigue siendo una decisión que debería implicar una discusión entre médico y paciente dada la necesidad de sopesar los beneficios cardiovasculares y del cáncer contra los riesgos hemorrágicos, las preferencias del paciente, el costo y otros factores"(2). 


\section{Coronary CT Angiography and 5-Year Risk of Myocardial Infarction: The SCOT-HEART Investigators}

Los pacientes con dolor torácico estable, sugestivo de enfermedad coronaria, pueden ser evaluados con una amplia variedad de estudios no invasivos. Aunque la angiografía coronaria por tomografía computada (CTA) mejora la certeza diagnóstica en la evaluación de pacientes con estas características, su efecto en los resultados a cinco años es desconocido ${ }^{(3)}$.

Con este fin, fue realizado el SCOT-HEART, estudio abierto, multicéntrico, grupo paralelo, en el que se aleatorizaron 4.146 pacientes (44\% sexo femenino, $11 \%$ diabéticos) con dolor torácico estable de bajo a moderado riesgo, referidos a una clínica cardiológica para evaluación, a tratamiento estándar y CTA (2.073 pacientes) o a tratamiento estándar solo (2.073 pacientes). El objetivo primario fue valorar muerte debida a enfermedad coronaria o IAM a cinco años ${ }^{(3)}$. Se excluyeron aquellos pacientes con insuficiencia renal, alergia al contraste, embarazo o síndrome coronario agudo en los últimos tres meses.

Los resultados fueron presentados por el Dr. David Newby y simultáneamente publicados en la revista The New England Journal of Medicine. A cinco años, el objetivo primario fue significativamente menor en el grupo asignado a CTA comparado con el tratamiento estándar solo (2,3\% [48 pacientes] vs 3,9\% [81 pacientes], HR0,59; IC95\%: $0,41-0,84 ; p=0,004)$. Aunque las tasas de angiografía coronaria invasiva y revascularización coronaria fueron más elevadas en los primeros meses de seguimiento en el grupo asignado a CTA y trata- miento estándar, en comparación con los que recibieron tratamiento estándar solo, las tasas generales fueron similares en ambos grupos a cinco años: la angiografía coronaria invasiva fue realizada en 491 pacientes en el grupo CTA, y en 502 pacientes en el grupo de tratamiento estándar (HR1,00; IC95\%: 0,88-1,13; p=0,993), y la revascularización coronaria fue realizada en 279 pacientes en el grupo de CTA vs 267 pacientes en el grupo de tratamiento estándar (HR1,07; IC95\%: 0,91-1,27; p=0,409). Se iniciaron más terapias preventivas y tratamientos antianginosos en los pacientes del grupo CTA. No hubo diferencias significativas entre los grupos en las tasas de muerte $\mathrm{CV}$, no $\mathrm{CV}$ o de cualquier cau$\mathrm{sa}^{(3)}$.

Con estos resultados, los autores concluyeron que el uso de CTA sumado al tratamiento estándar en pacientes con dolor torácico estable resulta en tasas significativamente más bajas de muerte debido a enfermedad coronaria o IAM no fatal a cinco años en comparación con el tratamiento estándar solo, sin diferencias significativas en las tasas de angiografía o revascularización coronaria ${ }^{(3)}$.

El Dr. Newby refirió que "la CTA es la prueba de elección no invasiva”. El autor puntualizó que sus resultados demostraron que la CTA "hizo una diferencia", añadiendo: "Muéstrenme otra prueba por imágenes que haya demostrado reducir las tasas de infarto de miocardio. No hay una. Entonces, ipor qué no la estamos utilizando como primera opción?"(4).

\section{Partial Oral Treatment of Endocarditis - POET}

Este estudio realiza un aporte significativo para la práctica clínica diaria, comprobando la no inferioridad de rotar la terapia intravenosa (IV) a la vía oral (VO) en pacientes con endocarditis infecciosa (EI) sobre válvulas cardíacas izquierdas ${ }^{(5)}$. Las guías de práctica clínica recomiendan tratamiento antibiótico IV por al menos 4 a 6 semanas cuando se trata una infección sobre las válvulas cardíacas izquierdas, ya sean nativas o protésicas ${ }^{(6,7)}$. Si bien la antibioticoterapia VO ha sido analizada en algunos estudios observacionales en situaciones clínicas específicas, ningún ensayo clínico había sido realizado previamente para validar el cambio a la $\mathrm{VO}^{(8,9)}$.

Presentado por el Dr. Henning Bundgaard, el estudio POET fue publicado simultáneamente en la revista The New England Journal of Medicine. Se trata de un ensayo clínico aleatorizado, multi- céntrico y sin cegamiento, llevado a cabo en Dinamarca. Se incluyeron 400 adultos con EI sobre válvulas izquierdas, clínicamente estables, con hemocultivos positivos a estreptococos, Enterococcus faecalis, Staphylococcus aureus o estafilococos coagulasa negativos. Los pacientes debían cumplir con los siguientes criterios: 18 años o más, diagnóstico por criterios de Duke, 10 días de tratamiento antibiótico IV apropiado y al menos una semana de tratamiento IV adecuado después de una cirugía valvular, temperatura menor a $38,0^{\circ} \mathrm{C}$ por más de dos días, caída de la proteína $\mathrm{C}$ reactiva al menos un $25 \%$ o un valor menor a $20 \mathrm{mg} / \mathrm{L}$, recuento de glóbulos blancos menor a $15 \times 10^{9} / \mathrm{L}$ y ausencia de abscesos por ecocardiografía. Todos debían contar con ecocardiografía transtorácica y transesofágica, realizadas dentro de las 48 horas de la aleatori- 
zación. Se excluyeron pacientes con índice de masa corporal mayor a 40, infección concomitante que requiriera terapia IV, incapacidad para consentir, sospecha de absorción oral reducida, o antecedentes de mala adherencia.

De los pacientes aleatorizados, 199 continuaron con tratamiento IV dirigido, mientras que 201 fueron asignados a realizar rotación a antibioticoterapia oral. Cuando era factible, el tratamiento se completaba de forma ambulatoria. Los regímenes orales fueron estandarizados por los autores y seleccionados según la susceptibilidad del microorganismo definida por concentración inhibitoria mínima. En todos los casos se realizó tratamiento combinado que incluyó: amoxicilina, ácido fusídico, linezolid, rifampicina, moxifloxacina y dicloxacilina. De los pacientes incluidos, $38 \%$ tuvo cirugía valvular y $27 \%$ válvula protésica infectada antes de la aleatorización, lo que indica que no se trató de una población de bajo riesgo estricto. Treinta y cinco pacientes tenían un dispositivo cardíaco implantado y la conducta con el dispositivo se tomó según decisión de un equipo multidisciplinario, retirándose en 14 pacientes.

El objetivo primario fue el compuesto de mortalidad por todas las causas, cirugía cardíaca no planificada, embolias o recaída de la bacteriemia a igual patógeno, desde el inicio del estudio hasta seis meses después de culminado el tratamiento. No hubo pérdidas durante el seguimiento. El resultado pri- mario ocurrió en el 9,0\% de los pacientes que recibieron antibioticoterapia VO versus $12,1 \%$ del grupo bajo tratamiento IV ( $\mathrm{p}=0,40)$, demostrando la no inferioridad. Respecto a los resultados secundarios, la incidencia de episodios embólicos, cirugía cardíaca no planificada y recaída de bacteriemia con el patógeno primario, fueron similares en los dos grupos. La mediana de la estancia hospitalaria tras la randomización fue de 19 días en el grupo tratado por vía IV y 3 días en el grupo tratado VO $(\mathrm{p}<0,001)$.

Durante la presentación los autores destacaron que la antibioticoterapia oral se puede administrar con seguridad durante la mitad del período de tratamiento antibiótico recomendado, potencialmente de forma ambulatoria, y que más de la mitad de los pacientes con EI podrían ser candidatos a recibir antibióticos orales. "Vimos resultados similares frente a una serie de comorbilidades, incluyendo si el paciente tenía una válvula nativa o prótesis y si se sometió a cirugía durante el curso de la enfermedad o si se trató de forma conservadora", resaltó el Dr. Henning Bundgaard durante su conferencia ${ }^{(10)}$.

A pesar de lo auspicioso del resultado, no se puede generalizar el abordaje para la infección con patógenos diferentes a los estudiados, resistentes o para infecciones por microorganismos desconocidos. El estudio presenta estrictos criterios de inclusión que deben tenerse en cuenta.

Ticagrelor plus aspirin for 1 month, followed by ticagrelor monotherapy for 23 months vs aspirin plus clopidogrel or ticagrelor for 12 months, followed by aspirin monotherapy for 12 months after implantation of a drug-eluting stent: GLOBAL LEADERS

GLOBAL LEADERS es un estudio de superioridad, aleatorizado y multicéntrico, efectuado en 131 centros de 18 países. Fue presentado en el auditorio principal del congreso por el profesor Patrick Serruys, y publicado de forma simultánea en la revista científica Lancet. Es hasta la fecha el único estudio que aleatorizó a los pacientes en el momento de la intervención coronaria percutánea, y que comparó dos estrategias de tratamiento antiplaquetario con un seguimiento de dos años ${ }^{(11)}$.

Este estudio fue diseñado para analizar los beneficios y riesgos del tratamiento con un potente antiagregante en combinación con aspirina en el primer mes, seguido de monoterapia con ticagrelor por 23 meses (grupo experimental 7.980 pacientes), en comparación con los esquemas de antiagregación actual de un año de doble terapia antiplaqueta- ria (DAPT) continuado por monoterapia con aspirina (grupo control 7.988 pacientes), tanto en síndrome coronario agudo (primeros 12 meses con ticagrelor y aspirina) como en enfermedad coronaria estable (primeros 12 meses con clopidogrel y aspirina) ${ }^{(11-13)}$.

El objetivo primario (mortalidad por cualquier causa o nuevo infarto con onda $Q$ ) en los primeros 12 meses fue significativamente menor en el grupo experimental que en el control (1,95\% vs 2,47\%, HR0,79; IC95\%: 0,64-0,98; $p=0,028)$, pero esta diferencia dejó de ser estadísticamente significativa a los 24 meses $(3,81 \%$ vs $4,37 \%$ HR0,87; IC95\%: $0,75-1,01 ; \mathrm{p}=0,073)$. Con respecto a estos hallazgos, Serruys explicó: "Si el estudio hubiera sido completado y terminado en un año, podría haber demostrado superioridad en el grupo experimental”. (14) Entre 
los resultados secundarios (mortalidad por cualquier causa, infarto de miocardio, trombosis definitiva del stent, y sangrado BARC [Bleeding Academic Research Consortium] grado 3 o 5) no existieron diferencias estadísticamente significativas entre los grupos. La adherencia en el segundo año fue $77,6 \%$ en el grupo experimental, mientras que en el grupo control alcanzó 93,1\%; la disnea fue la razón más común para el abandono del tratamiento en el grupo experimental en relación con el grupo control $(\mathrm{p}=0,005)^{(11,14-16)}$.

Uno de los investigadores del estudio, el Dr. Stephan Windecker, dijo que los resultados fueron decepcionantes: "Cuando tienes un estudio que no es positivo, hay un nivel de decepción. Si miras el hazard ratio y el valor $p$, si hubiésemos incluido más pacientes, habríamos cumplido el objetivo. Una de las restricciones fue el tamaño de la muestra. Si tuviéramos fondos suficientes, habríamos incluido hasta 20.000 pacientes. Esa es una explicación de por qué no tuvimos un estudio positivo"(17).

En vista de la alta tasa de abandono al tratamiento con ticagrelor, el aumento de la frecuencia de disnea, y el alto costo asociado al régimen experimental en relación con el grupo control, así como a la necesidad de su dosificación dos veces por día, el Dr. Deepak Bhatt, autor de la editorial del artículo en Lancet, considera que "la aspirina debería permanecer como la terapia antiplaquetaria preferida para prevención secundaria”(17,18).

\section{One-Year Outcomes after PCI Strategies in Cardiogenic Shock: CULPRIT-SHOCK}

La revascularización temprana mediante intervención coronaria percutánea (ICP) de la arteria responsable del infarto agudo de miocardio (IAM) con shock disminuye la mortalidad. En pacientes con IAM y enfermedad multivaso, varios estudios han demostrado evidencia a favor de la revascularización de las arterias no culpables previo al alta, incluso en términos de mortalidad cardiovascular, pero sin diferencias significativas en la mortalidad por todas las causas, como muestra un reciente metaanálisis ${ }^{(19,20)}$.

Sin embargo, en el contexto de IAM con shock cardiogénico existe poca evidencia acerca del tratamiento de las lesiones no culpables, ya que estos pacientes fueron excluidos de los estudios.

El año pasado fue publicado el CULPRITSHOCK trial, que evidenció que tras revascularizar la lesión culpable, continuar revascularizando otras lesiones en el evento índice empeora los resultados. El estudio mostró una disminución significativa del objetivo primario (el compuesto de muerte por cualquier causa e insuficiencia renal aguda que requirió terapia de sustitución renal) en el grupo con ICP del vaso culpable en comparación con la ICP inmediata multivaso a 30 días de la aleatorización. Dicho hallazgo se debió principalmente a una reducción significativa de las muertes por cualquier causa $(43,3 \%$ vs $51,6 \%$; HR0,84; IC95\%: 0,72-0,98; $p=0,03)$. Esto generó un cambio en la recomendación de las guías europeas con respecto a la revascularización de rutina de vasos no culpables en este grupo de pacientes, (pasando de ser IIa C en la guía de IAM con elevación del segmento ST de 2017 a III B en la reciente guía de revascularización del 2018)(19-22).
El pasado 25 de agosto, en la sesión Late-Breaking Science del Congreso, el Dr. Thiele presentó los resultados del estudio CULPRIT SHOCK a un año de seguimiento, reportados como los objetivos secundarios del estudio publicado en 2017: muerte por cualquier causa, terapia de sustitución renal, infarto recurrente, necesidad de nueva revascularización y rehospitalización por insuficiencia cardía$\mathrm{ca}^{(23)}$.

Este estudio comparó la revascularización mediante ICP de la lesión culpable del infarto (con opción a revascularización multivaso en etapas) versus la revascularización multivaso en el evento índice en pacientes con IAM y shock cardiogénico. Incluyó 706 pacientes con al menos dos lesiones $>70 \%$ en vasos $\geq 2 \mathrm{~mm}$ de diámetro, de los cuales a un año se evaluaron 684 pacientes. Se excluyeron los pacientes con resucitación mayor a 30 minutos, déficit cerebral severo, indicación de cirugía de revascularización, shock de causa mecánica o no cardiogénica, tromboembolismo pulmonar masivo, inicio del shock mayor a 12 horas previas a la aleatorización, mayores de 90 años, insuficiencia renal severa conocida con clearence de creatinina menor a $30 \mathrm{ml} / \mathrm{min}$, y expectativa de vida menor a seis meses. La mediana de edad fue 70 años ( $76 \%$ hombres). Se aleatorizaron en relación 1:1 a ambos grupos. La lesión culpable fue la primera en tratarse, implantándose stents liberadores de droga en más del 93\% de los pacientes. En el grupo en que se planificó durante el evento índice la ICP sólo del vaso culpable, hubo un cruzamiento de pacientes de $12,5 \%$ al grupo multivaso.Asimismo, en el grupo asignado a tratamiento multivaso, $9,4 \%$ recibió tratamiento sólo del vaso culpable. ${ }^{(23)}$. 
El estudio muestra una reducción no significativa de la mortalidad por todas las causas en el grupo ICP del vaso culpable (50,0\% vs 56,9\%, HR0,88; IC95\%: 0,76-1,01). Es muy importante recalcar que el análisis por protocolo y según como fueron finalmente tratados arrojó resultados similares. Tampoco hubo diferencias significativas en la mortalidad en el análisis por subgrupos, incluidos aquellos con oclusiones crónicas totales ${ }^{(23)}$.

Otro resultado interesante del ensayo clínico es el aumento del riesgo relativo de rehospitalización por insuficiencia cardíaca en el grupo ICP del vaso culpable (5,2\% vs 1,2\% RR4,46; IC95\%: $1,53-13,04)$ aunque "debe ser tomado con cautela debido a que este trabajo no tuvo el poder estadístico suficiente para demostrar este objetivo y la diferencia absoluta fue baja", puntualizó el Dr. Thiele a theheart.org|MedscapeCardiolog $y^{(24)}$.

"Este es un estudio extremadamente importante ya que nos ayuda en la práctica clínica”, señaló la panelista Julinda Mehilli al ser entrevistada. "La revascularización completa en el medio de la noche es realmente dificil y tener estos datos -que no necesitamos realizar esto (ICP multivaso) porque no tiene beneficio en la mortalidad- nos ayudará” agregó(24).

Por su parte, el Dr. S. James, uno de los coordinadores de las últimas guías europeas de IAM con elevación del segmento ST, en entrevista con theheart.org|MedscapeCardiology, mencionó: "La ICP inmediata multivaso puede justificarse si la lesión culpable es difícil de identificar o mal definida inicialmente, cuando varias lesiones culpables son identificadas, o en casos seleccionados en los cuales hay un vaso no culpable con limitación del flujo y estenosis severa irrigando una gran área miocárdi$c a^{\text {"(24). }}$.

Como conclusión, el investigador principal resaltó al final de la sesión que "los resultados a un año del CULPRIT-SHOCK apoyan el cambio reciente de la recomendación en la guía Europea”, pero no debemos olvidar que dicha actualización hace referencia al tratamiento rutinario de las lesiones no culpables y que la decisión final queda a criterio del hemodinamista ${ }^{(24)}$.

\section{Bibliografía}

1. Gaziano JM, Brotons C, Coppolecchia R, Cricelli C, Darius H, Gorelick PB, et al. use of aspirin to reduce risk of initial vascular events in patients at moderate risk cardiovascular disease (ARRIVE): a randomised double-blind, placebo-controlled trial. The Lancet 2018 Sep [consulta: 11 Oct 2018]; 392 (10152). Disponible en: https://doi.org/10.1016/ S0140-6736(18)31924-X
2. Highlights del Congreso Europeo de Cardiología (Munich 2018). Munich: IntraMed; 2018 [consulta: 15 Oct 2018]. Disponible en: https://www.intramed.net/contenidover.asp?contenidoID $=93003$ [Consulta: 8 de setiembre 2018].

3. Newby DE, Adamson PD, Berry C, Boon NA, Dweck MR, Flather M, et al. Coronary CT angiography and 5-year risk of myocardial infarction. $\mathrm{N}$ Engl J Med 2018;379 (10):924-33. doi: 10.1056/ NEJMoa1805971

4. Davenport L. SCOT-HEART: La angiotomografía computarizada coronaria se vinculó a menos muertes e infarto de miocardio no mortal, en el dolor torácico estable. New York: Medscape; 2018 [consulta: 13 Set 2018]. Disponible en: https://espanol.medscape.com/verarticulo/5903125

5. Iversen K, Ihlemann N, Gill SU, Madsen T, Elming H, Jenden KT, et al. Partial oral versus intravenous antibiotic treatment of endocarditis. N Engl J Med 2018. doi: 10.1056/NEJMoa1808312 2

6. Baddour LM, Wilson WR, Bayer AS, et al. Infective endocarditis in adults: diagnosis, antimicrobial therapy, and management of complications: a scientific statement for healthcare professionals from the American Heart Association. Circulation 2015; 132 (15): 1435-86. doi: 10.1161/CIR.0000000000000296

7. Habib G, Lancellotti P, Antunes MJ, Bongiorni MG, Casalta JP, Del Zotti F, et al. 2015 ESC guidelines for the management of infective endocarditis: the task force for the management of infective endocarditis of the European Society of Cardiology (ESC). Endorsed by: European Association for Cardio-Thoracic Surgery (EACTS), the European Association of Nuclear Medicine (EANM). Eur Heart J 2015; 36 (44): 3075-3128. doi: 10.1016/j.recesp.2015.11.015

8. Dworkin RJ, Lee BL, Sande MA, Chambers HF. Treatment of right-sided Staphylococcus aureus endocarditis in intravenous drug users with ciprofloxacin and rifampicin. Lancet 1989; 2: 1071-3. 17. doi: 10.1016/S0140-6736(89)91083-0

9. Iversen K, Høst N, Bruun NE, Elming H, Pump B, Christensen JJ, et al. Partial oral treatment of endocarditis. Am Heart J 2013; 165 (2):116-22. doi: 10.1016/j.ahj.2012.11.006

10. Wendling $\mathbf{P}$. Switch to oral antibiotics Safe in left-sided endocarditis, Slashes LOS [consulta: 3 Set 2018]. Munich: Medscape; 2018. Disponible en: https://www. medscape.com/viewarticle/901325 \#vp_2

11. Vranckx P, Valgimigli $\mathbf{M}$, Jüni $\mathbf{P}$, Hamm $\mathbf{C}$, Steg PG, Heg D, et al. Ticagrelor plus aspirin for 1 month, followed by ticagrelor monotherapy for 23 months vs aspirin plus clopidogrel or ticagrelor for 12 months, followed by aspirin monotherapy for 12 months after implantation of a drug-eluting stent: a multicentre, open-label, randomised superiority 
trial. Lancet 2018; 392(10151): 31858-0. doi: 10. 1016/S0140-6736(18)31858-0

12. Freixa X. Lo mejor de ESC 2018: estudio GLOBAL LEADERS. 2018 Ago 29 [consulta: 16 Set 2018]. En: Revista Española de Cardiología- Blog [Internet]. Madrid: SEC. 2018 [aprox. 2p.]. Disponible en: https://secardiologia.es/multimedia/blog/rec/9771lo-mejor-de-esc-18-estudio-global-leaders

13. Global Leaders [consulta: 16 Set 2018]. Rotterdam: European Cardiovascular Research Institute; 2018. Disponible en: https://www.ecri-trials.com/studies/ global-leaders/

14. Global Leaders trial: Long-term ticagrelor monotherapy after stenting does not improve outcomes vs standard dual antiplatelet therapy. ESC Congress news 2018 - Munich, Germany [Internet]. Sophia Antipolis Cedex: ESC; 2018 [Consulta 16 Set 2018]. Disponible en: https://www.escardio.org/Congresses-\&-Events/ ESC-Congress/Congress-resources/Congress-news/ global-leaders-trial-long-term-ticagrelor-monotherapy-after-stenting-does-not-improve-outcomes-vs-standard-dual-antiplatelet-therapy

15. Costabel J. GLOBAL LEADERS: un mes de aspirina y 24 meses de ticagrelor como estrategia para pacientes con angioplastia coronaria. 2018 Set 9 [Consulta:16 Set 2018]. En: Sociedad Interamericana de CardiologíaBlog [Internet]. Ciudad de México: SIAC [aprox. 1p.] Disponible en: http://www.siacardio.com/editoriales/ antiromboticos/global-leaders-un-mes-de-aspirina-y-24-meses-de-ticagrelor-como-estrategia-para-pacientes-con-angioplastia-coronaria/

16. ESC 2018 GLOBAL LEADERS: el ticagrelor como monoterapia no supera a los esquemas clásicos post angioplastia [Internet]. Buenos Aires: SOLACI; 2018 [Consulta 16 Set 2018]. Disponible en: https://solaci.org/es/2018/08/29/esc-2018-global-leaders-el-ticagrelor-como-monoterapia-no-supera-a-los-esquemas-clasicos-post-angioplastia/

17. O'Riordan M. GLOBAL LEADERS: ticagrelor monotherapy fails to beat conventional DAPT After PCI [Internet]. TCTMD, The Heart Beat; 2018 [consulta 16 Set 2018]. Disponible en: https://www.tctmd. com/news/global-leaders-ticagrelor-monotherapy-fails-beat-conventional-dapt-after-pci

18. Bhatt DL. Aspirin-still the GLOBAL LEADER in antiplatelet therapy. Lancet. 2018; 392 (10151): 896-7. doi: 10.1016/S0140-6736(18)31884-1.

19. Ibanez B, James S, Agewall S, Antunes MJ, Bucciarelli-Ducci C, Bueno H, et al. 2017 ESC Guidelines for the management of acute myocardial infarction in patients presenting with ST-segment elevation: The Task Force for the management of acute myocardial infarction in patients presenting with ST-segment elevation of the European Society of Cardiology (ESC). Eur Heart J 2018;39 (2):119-77. doi: 10.1093/eurheartj/ehx393

20. Bajraktari G, Jashari H, Ibrahimi P, Alfonso F, Jashari F, Ndrepepa G, et al. Complete revascularization for patients with ST-segment elevation myocardial infarction and multivessel coronary artery disease: a meta-analysis of randomized trials. Coron Artery Dis. 2018 ;29(3):204-15. doi: 10.1097/MCA. 0000000000000602 .

21. Thiele H, Akin I, Sandri M, Fuernau G, de Waha S, Meyer-Saraei R, et al. PCI strategies in patients with acute myocardial infarction and cardiogenic shock. N Engl J Med 2017; 377 (25): 2419-32. doi: 10.1056/NEJMoa1710261

22. Neumann F-J, Sousa-Uva M, Ahlsson A, Alfonso F, Banning AP, Benedetto U, et al. 2018 ESC/EACTS Guidelines on myocardial revascularization. Eur Heart J 2018;00:1-96 doi:10.1093/eurheartj/ehy394. [Epub ahead of print]

23. Thiele H, Akin I, Sandri M, de Waha-Thiele S, Meyer-Saraei R, Fuernau G, et al. One-Year Outcomes after PCI Strategies in Cardiogenic Shock. N Engl J Med. 2018 doi: 10.1056/NEJMoa1808788. [Epub ahead of print]

24. Wendling P. CULPRIT-SHOCK: benefits remain at 1 year for culprit-lesion-only PCI in MI with shock [consulta: 3 Set 2018]. Munich: Medscape; 2018. Disponible en https://www.medscape.com/viewarticle/ 901201 